\title{
Immunoglobulin Heavy Chain Complementarity Determining Region 3
}

National Cancer Institute

\section{Source}

National Cancer Institute. Immunoglobulin Heavy Chain Complementarity Determining

Region 3. NCI Thesaurus. Code C123154.

An amino acid sequence within the hypervariable region of an immunoglobulin heavy

chain that includes parts of the variable (V), diversity (D), and joining (J) regions. 